\title{
Tiny Face Detection And Recognition: A Review
}

\author{
Roshni Jambhulakar ${ }^{1}$ and Shailendra S. Aote ${ }^{2}$ \\ ${ }^{1,2}$ Shri Ramdeobaba College of Engineering \\ and Management, Nagpur, India
}

\section{ABSTRACT}

Face recognition is the method is most highly useful component for security surveillance with numerous biometrics and other surveillance systems, including several others states of art methods.Now a days have been made the object recognition one of the remaining open challenges in detecting small objects. Deep learning is used for tiny face recognitions.We explore so many aspects of the problem in this a finding a small faces. The role of small face is identifying by the small pixels of that image from the tiny faces. When we trying to find out the tiny faces, from lots of crowd. We need that image has the so many face objects. It means that image containing the large amount of data, while most recognition approaches in to be scale-invariant. The cues for recognizing 4 pixel tall faces are fundamentally different than, those for recognizing 400 pixels tall face.As we take out different approaches and train a separate detectors, for a different skills to maintain the efficiency detectors, are trained in multitask.Here is the deep learning week we used to make the features extracted from the multiple layers of a single feature hierarchy. While training a detected from large objects is strength forward the challenge, remaining train detectors for a small objects. We show that the context is a crucial and define templates that make us use of the massively large repetitive fields.We use the deep networks for preview the scale.

KEY WORDS: FACE RECOGNITION, TINY FACES, BIOMETRIC IDENTIFICATION, METHODS, APPLICATIONS, IMAGE PROCESSING, INDEPENDENT COMPONENT ANALYSIS, PRINCIPAL COMPONENT ANALYSIS, LINEAR DISCRIMINANT ANALYSIS, LINEAR REGRESSION ANALYSIS.

\section{INTRODUCTION}

In this world every person has his unique Identity, unique face features, and human identification process before differentiate from one person to other person by identifying their features.It has different facial characteristics nose, ears, forehead, eyes, lips etc.So face recognition method is used to identifying the people in images or in videos throughdetection, and then match within the independent components features.Facial recognition and other existing methods are used to

\section{ARTICLE INFORMATION}

*Corresponding Author: janbhulkarrv@rknec.edu

Received 18th Oct 2020 Accepted after revision 29th Dec 2020

Print ISSN: 0974-6455 Online ISSN: 2321-4007 CODEN: BBRCBA

Thomson Reuters ISI Web of Science Clarivate Analytics USA and Crossref Indexed Journal

\section{Clarivate
Analytics}

NAAS Journal Score 2020 (4.31)

A Society of Science and Nature Publication,

Bhopal India 2020. All rights reserved.

Online Contents Available at: http//www.bbrc.in/

Doi: http://dx.doi.org/10.21786/bbrc/13.14/48 identification procedure. But the problem occurs when we are trying to identify of thousands of tiny faces at a time. Now a day the tiny face identification, this topic is very popular in research area in worldwide from past few years.

Identifying the tiny faces from lots of crowd, sent work has done in object detection makes use of scale normalized classifierneed and reason classifiers. Run on the image which has the less scale pixels features. When resizing the reasons, we need to look for the size of the templates; we need to look for the size of the pixels of that image. So we can identify the tiny objects from that image itself. On other hand we need small templates so that can detect small faces also. While we're talking about the tiny face recognitions, we got so much of data so much of tiny faces. So we need to collect that large amount of data. Traininglarge collectionsof a scale specific detector, means suffer from lack of training data, for individual scale and inefficiency from running a number of large of detectives at test time.

$$
204
$$




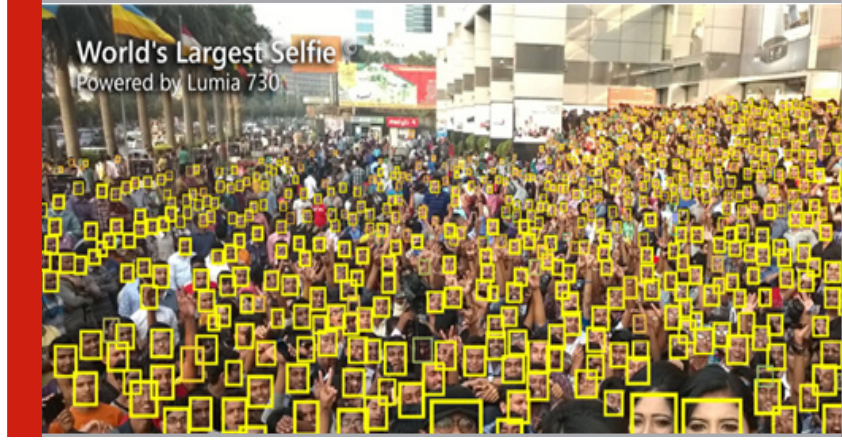

Facial recognition technology is becoming ubiquitous across the industries. It is considered as one of the fastest methods for image analysis, for human verification and image recognition through facial features and expressions. It involves extracting information, such as landmark, pose, expressions, gender, age, identity and etc.It has several applications including face biometrics for payments, security companies are using the facial recognition to secure their premises, immigration checkpoint use facial recognition to enforce smarter border control, fleet management companies can use face recognition to secure their vehicles, ride sharing companies can use facial recognition to ensure the right passengers are picked up by the right drivers, Iot benefits form facial recognition by allowing enhancement security measures and automatic access control at home, law enforcement can use facial recognition technologies as one part of their driven surveillance system, retailers can use facial recognition to customize offline offerings and to theoretical map online purchasing habits with their online ones.To identify a small object is a very challenge now days. We explore there more aspects of this problemin the context of face detection. The role is to find out the tiny faces from thousands of crowd and the task is when the object is of small scale but still it can be identified.

Literature Review: We give a brief overview of a recent works on different models of a face identification and recognition verification we discuss the recent face detection methods and applications and examples.

Previous Methods: Face Recognitions Methods: In the last decades there has been rapidly development of the reliable face recognition algorithms. The traditional face recognition algorithm can be categorized intovarious categories, holistic matching method, hybrid methods and feature based methods and that the holistic method group can be additional differentiate into a linear method and nonlinear projection methods.

Holistic matching methods: The holistic matching method is a complete all face feature is taken intoas an, input into a face features catching system.Holistic methods are Eigen facesapplications have given avarious good result of nonlinear projections method, appearance is based method search is a independent component analysis (ICA) and linear regression classifier (LRC), linear discriminant analysis (LDA) and principal component analysis(PCA).
In the starting stage of 1970 , The face recognition method was called as a 2D pattern Face recognition method problem. The distance of between in precious points isa used to recognized known feature of faces; example calculating thegiven distance between the face features like eyes,head, nose or other important points for measuring various angles of various facial components of faces. But it is verynecessary that the face identification and face recognition method systems to be automatic face recognition on features are such a challenging job now days. It important problem that is has attracted for research towards face recognition, If they have various background psychology patterns recognition, neural network as computer visions and different computer graphics security biometrics etc.

Figure 2: Face Recognition System

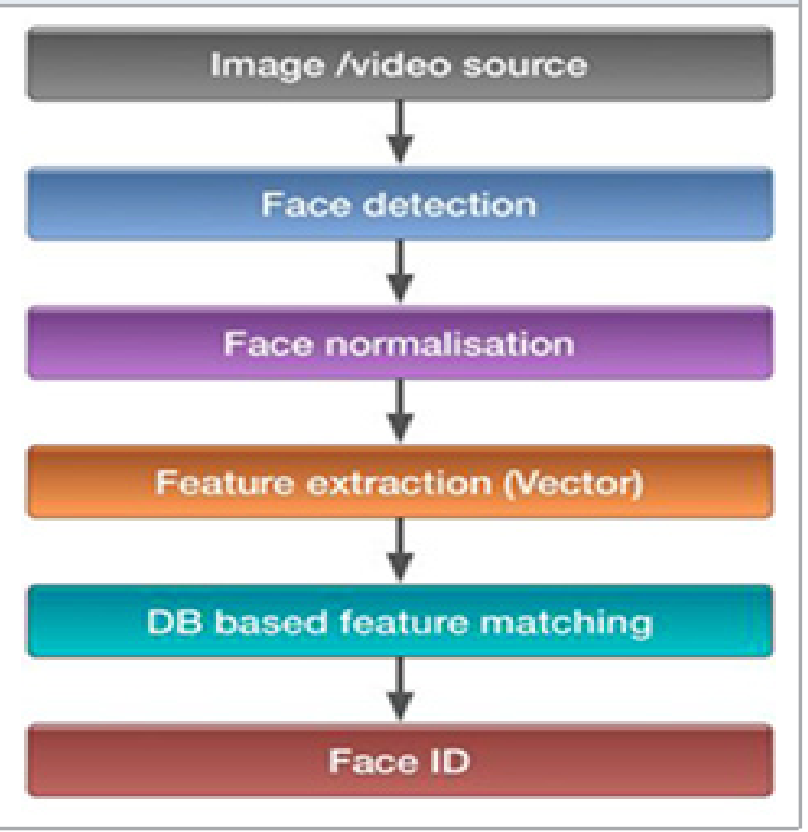

Due to large variations in conditions facial expressions and other factors this method may fail to adequately represent the faces. The main reason is that the face white and lie on a complex nonlinear and non-convex main fold in the high dimensional spaces. In this order to delay with such a case is known in linear extensions, have been propose like a kernel PCA(KPCA), kernel LDE (KLDE). Do most nonlinear methods using the Kernel techniques, the general idea consists of Mac paying the input face images into a higher dimensional space. In which the main food of the face is linear and simplified so the traditional linear methods can be applied.

In the example of the iron faces the flowchart contain stages to classify the problem in given figure, the different stages are illustrates; in the beginning stage are to add different set of the images into a given database. These different input images are named asainput training sets of face recognition and this is because they will be used when we used to be compared thedifferent images and when we create the Eigen faces as an input. II stage is to 
create the Eigen faces of the other input image. It meansis extracting characteristics face features from ainput image. The two different input imageare differently normalized to each line of a nose, eyes, forehead those are then resize, so that those features have the same distanceof Eigen faces, so can that extracted from the input image various dataas using the arithmetical tools it is principal component analysis(PCA). When input image Eigen faceshas been created input image will be elected by as vector of weighted objects.

So now those systems are ready to accept the entire query is the input object weight of that incomingother input image is presented and then compared that with two different images to the weighted objects of those are sorted alreadyis given in the system. If the image weights are given as 4 solved for comparison. It is used to be considered as an identified the identification of the input image is done at the stage identification by the finding the various images in the database, which is weight, are very nearest tothe input image in identifications. In the given database of input images with the clauses will be prepared as a heat to the current user of the face recognition system as the sorted image.

Eigen Faces Feature based Methods: In Eigen faces feature based methods, the basic features of face such as forehead, eyes, nose, ears, chicks etc. are the first of all extracted from the input image and their locations of features and local statistics of face recognition are feed into a structural classifier of features. Now a day'schallenges for feature extraction method are a given features Restoration. This method is when the face recognition system tries to retrieve different features from the input imagethose are differently invisible due to a large different variations of image e.g. When we match the frontal view of image with the given profile image. We found out the similar features sometime. General dimensionality reduction techniques are used such as independent component analysis, ISO map, kernel PCA, latent semantic analysis, partial least squares, principal component analysis, multifactor dimensionality reduction, nonlinear dimensionality reduction, multilinear principal component analysis, multilinear subspace learning, Semidefinite embedding, auto encoder, generic methods which is based on edges of image, shape, size, line and curve features of different views template are based methods of structural view matching method takes into a considered ofsymmetricalconstructions on the different features on input images.

Hybrid Methods: Do hybrids faces recognition methods, used the various combination ofholistic method and different feature extraction methods. Generally when we include the hybrid we use the 3D images in the hybrid method generally, the image is the form of the 3D the object in that image is that object looks like as and 3Dthis system is trying to understand the curves the position of that object. Suppose if the four head of that object is tilted towards to the left so that system, will detect of image that object by their particular shape. Even a various faces in system wouldgives because, these system use death as an existence on measurements of given images which gave that detailed information to construct a over all facefeatures extracted in the 3D system proceed does Measurement,Detections, Representation, PositionsMatching of face features.

Measurements - Given the measurement to that the object like the object is having the template of specific faces on the particular object inside in that image,like if we want to focuses on face so it gives the template of that the shape and size of the eyes and the angle of nose.Detection - Image scanning and capturing the exact object from that real time image. Representation - In the image convert that image into a code, we convert that image into a numerical representation of that prescribe object. Position- Considering the location of the damage, the angle of that image the size of the image and the curve which occur in that image for the real time object. Matching- Comparing the existing image of object which the received image of object from the database.

Figure 3: Flow Chart of the Eigen faces

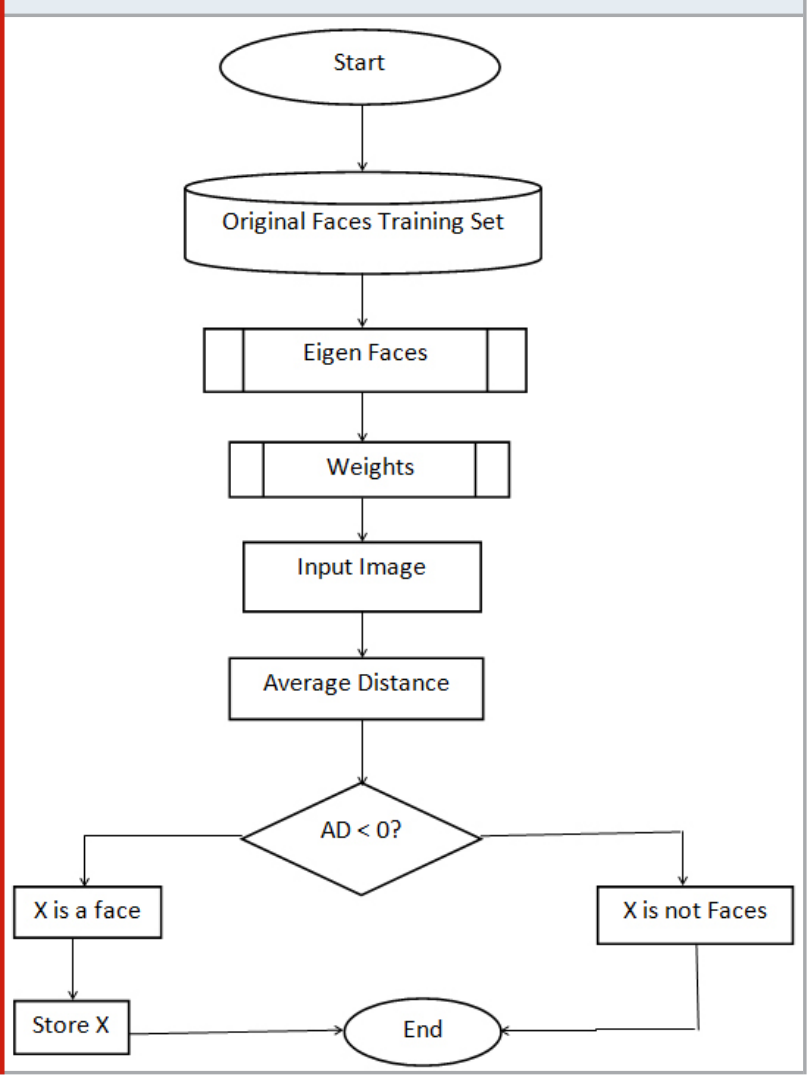

\section{CONCLUSION}

In recent days so many researchers are doing research in Tinny face recognition and now on from many years, in face recognition area is the one of the challenging area for the research. In this paper we have the concept of the face recognition methods given and existing work which has done it in the face recognition method. In this paper the review information and the understanding about Tinny face recognition methods are provide for 
readers for better understand. In the future research large scale Tinny face recognition applications such as the E-Commerce, student id in colleges for identifying the tiny faces or even personal identityit is a challenging task now days in the face recognitions methods and in recent years this topic is having high probability for future research.

\section{REFERENCES}

HAO WU, KE ZHANG , AND GUOHUI TIAN, “Simultaneous Face Detection and Pose EstimationUsing Convolutional Neural Network Cascade

School of Control Science and Engineering, Shandong University, Jinan 250061, ChinaCorresponding author: Ke Zhang (sdu_kezhang@163.com)

Peiyun Hu, Deva Ramanan, “Finding Tiny Faces” Robotics InstituteCarnegie Mellon Universityfpeiyunh,devag@ cs.cmu.edu.

RajeevRanjan, Ankan Bansal, Jingxiao Zheng, Hongyu $\mathrm{Xu}$, Joshua Gleason, Boyu Lu, AnirudhNanduri,JunCheng Chen, Carlos D. Castillo, Rama Chellappa,
“JOURNAL OF LATEX CLASS FILES, VOL. 14, NO. 8, AUGUST 20151 A Fast and Accurate System for Face Detection,Identification, and Verification

SHI LUO , XIONGFEI LI , (Member, IEEE), RUI ZHU , AND XIAOLI ZHANG, "Small Faces Attention Face Detector" Key Laboratory of Symbolic Computation and Knowledge Engineering of Ministry of Education, Jilin University, Changchun 130012, ChinaCollege of Computer Science and Technology, Jilin University, Changchun 130012, ChinaCorresponding author: Xiaoli Zhang (xiaolizhang@jlu.edu.cn)

Wenqi Wu, Yingjie Yin, Xingang Wang, and De Xu , Senior Member, IEEE. "Face Detection With Different ScalesBased on Faster R-CNN “

Xiangyu Zhu, Zhen Lei, Xiaoming Liu, Hailin Shi, Stan Z. Li, "Face Alignment Across Large Poses: A 3D Solution 1Institute of Automation, Chinese Academy of Sciences2Department of Computer Science and Engineering, Michigan State Universityfxiangyu. zhu,zlei,hailin.shi,szlig@nlpr.ia.ac.cnliuxm@msu.edu 